\section{Ecuaciones de predicción del gasto de energía en reposo en escolares de la Ciudad de México}

\section{RESUMEN}

Introducción: existen diferentes ecuaciones de predicción para estimar el gasto de energía en reposo en niños; sin embargo, no hay consenso de cuál es la más adecuada para usarse en población pediátrica mexicana.

Objetivo: conocer la concordancia del gasto de energía en reposo medido por calorimetría indirecta con el estimado con diferentes ecuaciones de predicción en un grupo de escolares de la Ciudad de México.

Material y método: se realizó un estudio transversal en escolares de 6 a 12 años de edad $(n=63)$. El gasto de energía en reposo fue medido mediante calorimetría indirecta, se usó como referencia para conocer la exactitud de las ecuaciones de predicción de Schofield, de FAO/OMS, de Tverskaya y de Harris-Benedict. Para conocer la concordancia entre las ecuaciones y el gasto de energía en reposo se estimaron coeficientes de correlación intraclase $(r)$.

Resultados: en esta población las cuatro ecuaciones sobrestimaron el gasto de energía en reposo; en promedio, las diferencias fueron de 12.1 a $97.5 \mathrm{kcal} /$ día. Las ecuaciones que tuvieron la mayor concordancia con el gasto de energía en reposo medido fueron las de Schofield y las de FAO/OMS $(r=0.74)$, mientras que las de Tverskaya y de Harris-Benedict tuvieron la menor $(r=0.64)$. La concordancia fue menor en los niños más pequeños, especialmente con las ecuaciones de Harris-Benedict $(r=0.33)$ y Tverskaya $(r=0.47)$.

Conclusiones: aunque se requiere más investigación sobre la conveniencia de usar ecuaciones de predicción del gasto de energía en reposo en población pediátrica de México, nuestros resultados muestran que las ecuaciones de FAO/OMS o las de Schofield son las más adecuadas.

Palabras clave: gasto de energía en reposo, calorimetría indirecta, escolares.

\section{Equations to estimate resting energy expenditure in school children from Mexico City. A diagnostic accuracy study}

\section{ABSTRACT}

Introduction: There are different prediction equations for estimating resting energy expenditure (REE) in children. However, there is not consensus on which it is the most appropriate for use in Mexican pediatric population.
Marlu Estephanie Becerril-Sánchez ${ }^{1}$

Margarita Flores-Reyes ${ }^{1}$

Norma Ramos-Ibáñez ${ }^{2}$

Luis Ortiz-Hernández ${ }^{2}$

${ }^{1}$ Asistente de investigación del Departamento de Atención a la Salud.

${ }^{2}$ Profesor(a) del Departamento de Atención a la Salud.

Universidad Autónoma Metropolitana Unidad Xochimilco.
Recibido: 4 de noviembre del 2014

Aceptado: 14 de abril del 2015

Correspondencia: Luis Ortiz-Hernández DAS-UAM

Calzada del Hueso 1100

CP 04960 México, D.F.

Tel.: 54-83-7243

lortiz@correo.xoc.uam.mx

Este artículo debe citarse como

Becerril-Sánchez ME, Flores-Reyes M, Ramos-Ibáñez $\mathrm{N}$, Ortiz-Hernández L. Ecuaciones de predicción del gasto de energía en reposo en escolares de la Ciudad de México. Acta Pediatr Mex 2015;36:147-157. 
Objective: To know the correlation of REE measured by indirect calorimetry with different estimates from prediction equations in a group of school children from Mexico City.

Material and method: A cross-sectional study with schoolchildren aged 6 to 12 years $(n=63)$ was carried out. REE was measured by indirect calorimetry, which was used as reference to assess the accuracy of the prediction equations of Schofield, FAO/WHO, Tverskaya and HarrisBenedict. To assess the agreement between measured TEE and prediction equations, intra-class correlation coefficients $(r)$ were estimated.

Results: In the total population the four equations overestimated REE, average differences were from 12.1 a $97.5 \mathrm{kcal}$. The equations that had the highest agreement with the measured TEE were Schofield and FAO/ WHO $(r=0.74)$, whereas Tverskaya and Harris-Benedict had the lowest $(r=0.64)$. Agreement was lower in younger children, especially with the equations of Harris-Benedict $(r=0.33)$ and Tverskaya $(r=0.47)$.

Conclusions: Although it is required more research on the convenience of using prediction equations of TEE in the Mexican pediatric population, our results showed that the equations of FAO/WHO or Schofield are more appropriate.

Key words: resting energy expenditure, indirect calorimetry, schoolchildren.

\section{INTRODUCCIÓN}

El sobrepeso y la obesidad son problemas frecuentes en los escolares mexicanos. ${ }^{1}$ Esto evidencia la necesidad de adoptar medidas para prevenir y tratar la ganancia excesiva de peso en la población pediátrica de México. El balance energético de un individuo depende de su ingesta y de su gasto energético; los desequilibrios en este balance pueden producir ganancia o pérdida de peso. ${ }^{2}$ Para que se logre una mejora en el estado nutricional de los escolares es necesario que se establezcan acertadamente sus requerimientos o gasto de energía, para no sobre- o subestimar sus necesidades y con ello asegurar que su nutrición y crecimiento sean adecuados.

Existen varios métodos para calcular el gasto energético de un individuo: calorimetría directa e indirecta, el agua doblemente marcada, el mo- nitoreo de frecuencia cardiaca y las ecuaciones predictivas. Sin embargo, con excepción de las últimas, el costo de los equipos requeridos impide tener una estimación precisa de las necesidades energéticas. Además, en ocasiones, incluso los métodos más económicos (e.g. calorimetría indirecta o monitoreo de frecuencia cardiaca) no son factibles de utilizar en la práctica clínica pues implican tiempo, compromiso y algunas incomodidades para los sujetos en evaluación. Por ello, una alternativa son las ecuaciones predictivas cuyo uso es simple, sin costo e implica actividades mínimas tanto del personal de la salud como del individuo.

Actualmente, se utilizan varias ecuaciones predictivas para calcular el gasto de energía en reposo en niños (Cuadro 1). Las primeras ecuaciones de predicción para calcular el gasto de energía en reposo en población infantil fueron 
Cuadro 1. Ecuaciones de predicción del gasto de energía en reposo

\begin{tabular}{|c|c|c|c|}
\hline Autor & Sexo & Edad (años) & Ecuación de predicción \\
\hline \multirow[t]{2}{*}{ Harris-Benedict $^{7}$} & Hombres & $16-91$ & $66.4730+(13.7516 \times P)+(5.0033 \times E)-(6.7550 \times \text { edad })^{a}$ \\
\hline & Mujeres & $15-88$ & $665.0955+(9.5634 \times \mathrm{P})+(1.8449 \times \mathrm{E})-(4.6756 \times \text { edad })^{\mathrm{a}}$ \\
\hline \multirow[t]{4}{*}{ Schofield con peso ${ }^{3}$} & Hombres & $3-10$ & $(19.6 \times P)+(130.3 \times E)+414.9^{b}$ \\
\hline & & $10-18$ & $(16.25 \times P)+(137.2 \times E)+515.5^{b}$ \\
\hline & Mujeres & $3-10$ & $(16.97 \times P)+(161.8 \times E)+371.2^{b}$ \\
\hline & & $10-18$ & $(8.365 \times P)+(465 \times E)+200^{b}$ \\
\hline \multirow[t]{4}{*}{ Schofield con peso y estatura ${ }^{3}$} & Hombres & $3-10$ & $(22.706 \times P)+504.3^{b}$ \\
\hline & & $10-18$ & $(17.686 \times P)+658.2^{b}$ \\
\hline & Mujeres & $3-10$ & $(20.315 \times P)+485.9^{b}$ \\
\hline & & $10-18$ & $(13.384 \times P)+692.6^{b}$ \\
\hline \multirow[t]{4}{*}{$\mathrm{FAO} / \mathrm{OMS}^{5}$} & Hombres & $3-10$ & $22.7 P+495^{b}$ \\
\hline & & $10-18$ & $17.5 \mathrm{P}+651^{\mathrm{b}}$ \\
\hline & Mujeres & $3-10$ & $22.5 P+499^{b}$ \\
\hline & & $10-18$ & $12.2 P+746^{b}$ \\
\hline \multirow[t]{2}{*}{ Tverskaya $^{6}$} & Hombres & $6-18$ & $775+(28.4 \times$ MLG $)-(37 \times$ edad $)+(3.3 \times$ MG $)+(82 \times 1)^{c}$ \\
\hline & Mujeres & $6-18$ & $775+(28.4 \times$ MLG $)-(37 \times$ edad $)+(3.3 \times M G)+(82 \times 0)^{c}$ \\
\hline
\end{tabular}

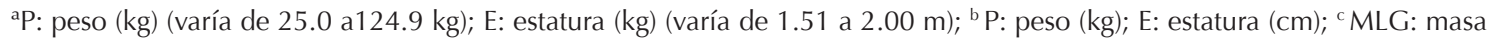
libre de grasa (kg); MG: masa grasa $(\mathrm{kg})$.

creadas en 1985 por Schofield; ${ }^{3}$ éstas consistieron en una serie de ecuaciones lineales que predecían el gasto de energía en reposo por sexo y grupo de edad, dentro de los que se incluían los grupos de escolares (3 a 10 años) y adolescentes (10 a 18 años), a partir del peso (Schofield-P) o de la combinación de la estatura y el peso (Schofield-PE). La inexactitud de éstas oscila entre 7 y $10 \%{ }^{4}$ En el mismo año la Organización de las Naciones Unidas para la Alimentación y la Agricultura con la Organización Mundial de la salud (FAO/OMS) ${ }^{5}$ publicaron sus ecuaciones para estimar el gasto de energía en reposo a partir de la información sobre sexo, edad y peso. Posteriormente, Tverskaya y sus colaboradores ${ }^{6}$ desarrollaron ecuaciones predictivas incluyendo individuos con peso normal y con sobrepeso u obesidad.

El uso de las ecuaciones predictivas existentes está limitado por el hecho de que algunas de ellas $^{6,7}$ fueron desarrolladas con individuos residentes de países industrializados, cuyo ambiente y características físicas (e.g. estatura o grado de adiposidad) pueden diferir sustancialmente. Con excepciones, ${ }^{6}$ las ecuaciones predictivas fueron desarrolladas con muestras en las que predominaban los niños de peso y estatura normales, por lo cual se desconoce si son aplicables a niños con obesidad o desnutrición crónica. Actualmente persiste la controversia de cuál es la ecuación predictiva más apropiada para calcular el gasto de energía en reposo en niños mexicanos.

\section{OBJETIVO}

El objetivo del presente estudio fue conocer la concordancia del gasto de energía en reposo medido mediante calorimetría indirecta con el gasto de energía en reposo estimado con las diferentes ecuaciones predictivas en un grupo de escolares de la Ciudad de México.

\section{MATERIAL Y MÉTODO}

Estudio transversal con 63 escolares de sexo indistinto cuyas edades oscilaron entre 6 y 12 
años de edad y que estudiaban en una escuela primaria ubicada en la Delegación Coyoacán, Distrito Federal. Se incluyeron todos los niños de los que se obtuvo el consentimiento informado y firmado por sus padres o tutores autorizando y sin ningún problema de salud. Se excluyeron los escolares cuyo resultado en la calorimetría en reposo no fue satisfactorio debido a que su respiración no se mantuvo en los límites adecuados o no fue detectada por el calorímetro por más de 5 minutos $(\mathrm{n}=2)$.

La calorimetría indirecta es el método estándar para la medición del gasto de energía en reposo. ${ }^{8}$ El gasto de energía se estima a partir del consumo de oxígeno $\left(\mathrm{O}_{2}\right)$ y la producción de bióxido de carbono $\left(\mathrm{CO}_{2}\right)$. Siguiendo procedimientos estandarizados ${ }^{9,10}$ el $\mathrm{VO}_{2}$ y el $\mathrm{VCO}_{2}$ fueron medidos con un equipo de calorimetría indirecta marca Cardio Coach, calibrado una sola vez por la mañana antes de las evaluaciones. Los niños fueron citados con ropa deportiva y tenis, sin haber ingerido alimentos por lo menos 3 horas antes de la evaluación. Cada niño fue medido una sólo ocasión por la mañana. Antes de cada medición los niños permanecían sentados en reposo por lo menos 20 minutos. La medición del intercambio de gases se realizó por un periodo aproximado de 12 minutos, durante el cual los niños estuvieron sentados, despiertos, sin realizar ningún movimiento que involucrara esfuerzo físico, lo más tranquilos posible, viendo una película y respirando a través de la mascarilla. Las mediciones las realizaron dos pasantes de la licenciatura en nutrición capacitadas en el manejo del equipo siguiendo protocolos estandarizados ${ }^{10}$ y las indicaciones del fabricante. Para garantizar la calidad de las mediciones la medición de gases se terminó cuando el coeficiente de variación fue menor a $10 \%$ durante un lapso de 5 minutos..$^{10} \mathrm{El}$ gasto de energía en reposo se obtuvo utilizando los valores de $\mathrm{VO}_{2}$ y $\mathrm{VCO}_{2}$ medidos utilizando la ecuación abreviada de Weir: ${ }^{11}$ gasto de energía en reposo $=3.9 \times \mathrm{VO}_{2}+1.1 \times \mathrm{VCO}_{2}$
El gasto de energía en reposo fue estimado usando las ecuaciones predictivas de Schofield (Schofield-peso y Schofield-peso/estatura), ${ }^{3}$ de la FAO/OMS ${ }^{5}$ y de Tverskaya $^{6}$ (Cuadro 1). A pesar de que fueron desarrolladas con población adulta, se decidió evaluar las ecuaciones predictivas de Harris-Benedict ${ }^{7}$ porque son utilizadas con frecuencia en población pediátrica.

Se realizaron mediciones antropométricas siguiendo los procedimientos descritos por Lohman y sus colaboradores. ${ }^{12}$ Antes de iniciar el trabajo de campo las observadoras fueron capacitadas de acuerdo con los procedimientos descritos por Habicht. ${ }^{13}$ Para medir el peso se utilizó una báscula marca SECA, digital, portátil, con precisión de $0.1 \mathrm{~kg}$. La estatura fue medida con estadímetro portátil marca SECA con precisión de $0.1 \mathrm{~cm}$. Los pliegues subcutáneos fueron medidos con un plicómetro Harpenden con precisión de $0.2 \mathrm{~mm}$.

A partir del peso y la estatura se calculó el índice de masa corporal $\left(\mathrm{IMC}=\right.$ peso/estatura $\left.{ }^{2}\right)$. Utilizando las tablas de la Organización Mundial de la Salud ${ }^{14}$ se estimó la puntuación Z del índice de masa corporal para edad. Los puntos de corte para este índice fueron: a) peso normal: $\leq 0.99$ y b) sobrepeso y obesidad: $\geq 1.00$. Con las mismas referencias ${ }^{14}$ también se estimó la puntuación $Z$ de estatura para edad utilizando las tablas de la Organización Mundial de la Salud con la siguiente clasificación: a) estatura baja: $\leq-1.00$ y b) estatura normal: $\geq-1.01$.

El porcentaje de grasa corporal se calculó con la ecuación de Slaughter y sus colegas..$^{15}$ Cuando la suma del pliegue subcutáneo tricipital y el subescapular fueron menores a $35 \mathrm{~mm}$ las ecuaciones utilizadas son:

Mujeres:

1.33 ( $\sum$ de pliegues $)-0.013\left(\sum \text { de pliegues }\right)^{2}-2.5$ 
Varones:

1.21 ( $\sum$ de pliegues $)-0.008\left(\sum \text { de pliegues }\right)^{2}+I^{*}$

Donde:

$I^{*}=$ valor de acuerdo con la etapa de maduración sexual del niño (prepuber: -1.7; puber: -3.4; pospuber: -5.5). La maduración sexual fue determinada siguiendo el procedimiento descrito por Mirwald y su grupo. ${ }^{16}$

Cuando la suma del pliegue subcutáneo tricipital y el subescapular fue igual o mayor a $35 \mathrm{~mm}$ las ecuaciones son:

$$
\begin{aligned}
& \text { Varones }=0.783\left(\sum \text { de pliegues }\right)+1.6 \\
& \text { Mujeres }=0.546\left(\sum \text { pliegues }\right)+9.7
\end{aligned}
$$

Se consideró que un niño tenía obesidad cuando su porcentaje de grasa corporal fue igual o mayor a $25.0 \%$, mientras que el punto de corte en las niñas fue $30.0 \% .^{17}$

Respecto al análisis estadístico, se evaluó la distribución de las diferentes estimaciones de gasto de energía en reposo y se observó que tenían una distribución sesgada (Figura 1) ya que todas tuvieron un valor de sesgo mayor a 1.00. Sólo el gasto de energía en reposo medido tuvo una distribución simétrica. Por ello, se estimaron medianas del gasto de energía en reposo medido y calculado con las ecuaciones predictivas. Para evaluar si existían diferencias entre ecuaciones se usó la prueba de Wilcoxon. Se utilizó la prueba de $U$ de Mann-Whitney para muestras independientes para comparar la media de dos grupos; mientras que para la comparación de tres grupos se usó la prueba de Kruskal-Wallis. Para evaluar la concordancia entre las ecuaciones predictivas y la medición por calorimetría se estimaron coeficientes de correlación intraclase. Las diferencias y correlaciones fueron consideradas estadísticamente significativas cuando $p<0.05$. El análisis estadístico se realizó con el programa SPSS versión 15.0.

\section{RESULTADOS}

El trabajo de campo fue realizado en los meses de enero y febrero del 2011. Durante las evaluaciones no se identificó ningún incidente o efecto adverso. El Cuadro 2 incluye las características de la población estudiada. Las mujeres fueron más de la mitad de la población (61.9\%). La distribución por edades fue homogénea ya que casi un tercio de la población se ubicó en cada grupo (rango de 6 a 12 años). En cuanto al estado nutricional uno de cada diez niños tuvo estatura baja, mientras que la tercera parte de la población tenía sobrepeso u obesidad. De acuerdo con la evaluación del porcentaje de grasa corporal, una cuarta parte de la población tenía obesidad.

Las medianas de gasto de energía en reposo medido y del gasto de energía en reposo estimado con las ecuaciones predictivas se presentan en el Cuadro 3 y los histogramas de cada variable en la Figura 1. Las ecuaciones predictivas proporcionaron, sistemáticamente, estimaciones superiores a la medición del gasto de energía en reposo por calorimetría; sin embargo, no existieron datos atípicos. En la población total el rango de sobrestimación de las ecuaciones predictivas fue de 12.1 a $97.5 \mathrm{kcal} /$ día, siendo las diferencias estadísticamente significativas con las ecuaciones de la FAO/OMS (diferencia de 52.1 respecto al gasto de energía en reposo medido) y la de Tverskaya y su grupo (diferencia de $97.5 \mathrm{kcal} /$ día). De igual modo, la distribución de las estimaciones producidas con las ecuaciones predictivas tuvieron una distribución desplazada hacia la derecha respecto a la distribución del gasto de energía en reposo medido (Figura 1).

El promedio de gasto de energía en reposo calculado con Harris-Benedict fue el que más se acercó 


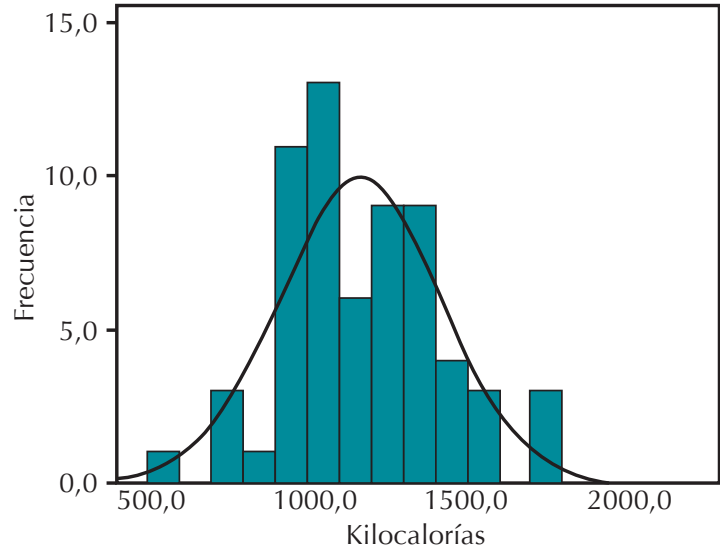

1a. Gasto medido por calorimetría

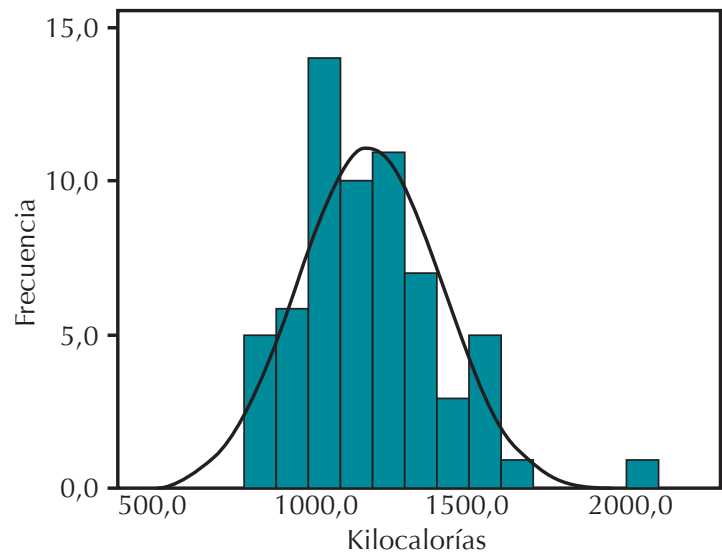

1c. Gasto estimado por ecuaciones de Schofield con peso

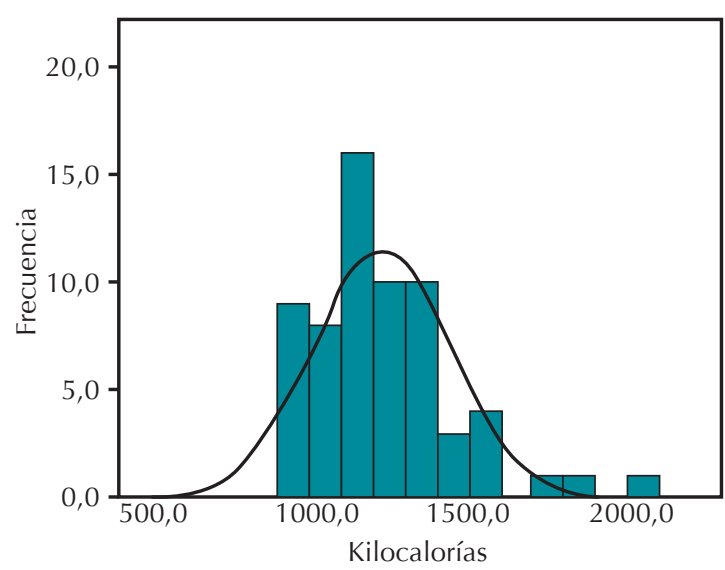

1e. Gasto estimado por ecuaciones de FAO/OMS

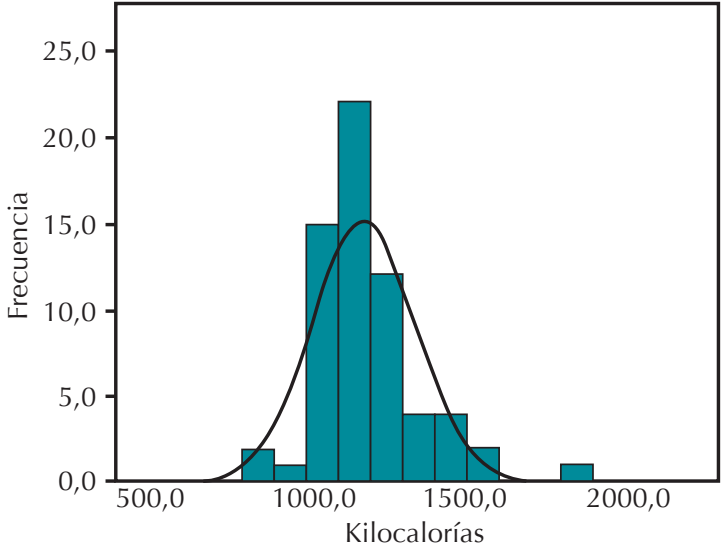

1b. Gasto estimado por ecuaciones de Harris-Benedict

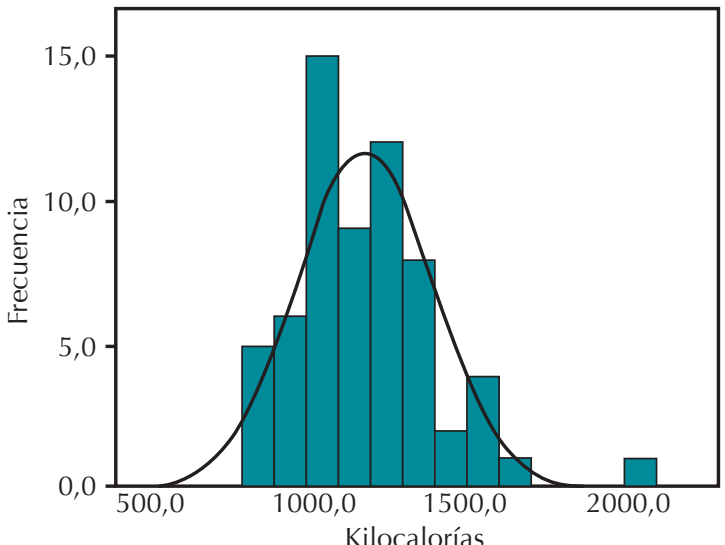

1d. Gasto estimado por ecuaciones de Schofield con peso y estructura

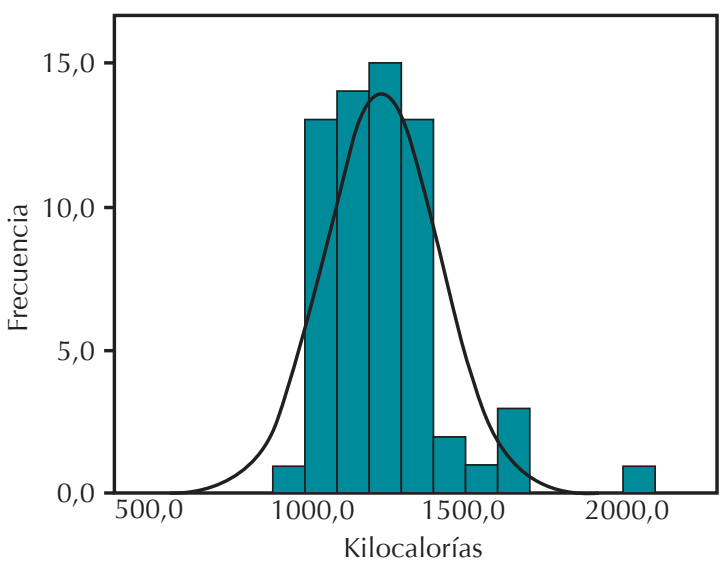

1f. Gasto estimado por ecuaciones de Tverskaya

Figura 1. Histogramas del gasto de energía en reposo medido y estimado por ecuaciones de predicción. 
Cuadro 2. Características de la población estudiada

\begin{tabular}{|c|c|c|}
\hline & $\mathbf{N}$ & $\%$ \\
\hline Total & 63 & 100 \\
\hline \multicolumn{3}{|l|}{ Sexo } \\
\hline Hombres & 24 & 38.1 \\
\hline Mujeres & 39 & 61.9 \\
\hline \multicolumn{3}{|l|}{ Edad (años) } \\
\hline $6-7$ & 21 & 33.3 \\
\hline $8-10$ & 22 & 34.9 \\
\hline $11-12$ & 20 & 31.7 \\
\hline \multicolumn{3}{|l|}{ Estatura } \\
\hline Ваја & 7 & 11.1 \\
\hline Normal & 56 & 88.9 \\
\hline \multicolumn{3}{|l|}{ Índice de masa corporal } \\
\hline Normal & 44 & 69.8 \\
\hline Sobrepeso u obesidad & 19 & 30.2 \\
\hline \multicolumn{3}{|l|}{$\%$ grasa corporal } \\
\hline Normal & 47 & 74.6 \\
\hline Obesidad & 16 & 25.4 \\
\hline
\end{tabular}

al promedio del gasto de energía en reposo medido, con una diferencia de $12.1 \mathrm{kcal} /$ día. Respecto a las diferencias entre sexos el gasto de energía en reposo estimado fue más alto en los niños que en las niñas. En los hombres existieron diferencias estadísticamente significativas del gasto de energía en reposo medido con las ecuaciones predictivas de Schofield-peso, Schofield-peso/ estatura y Tverskaya y sus colaboradores (66.4, 53.1 y $52.1 \mathrm{kcal} / \mathrm{día}$ de diferencia respecto al gasto de energía en reposo medido, respectivamente). En las mujeres las ecuaciones predictivas de FAO/OMS y deTverskaya y su grupo tendieron a producir valores mayores al gasto de energía en reposo medido (58.2 y $56.8 \mathrm{kcal} / \mathrm{día})$.

Cuadro 3. Medianas (rango intercuartil) del gasto energético en reposo medido y calculado con varias ecuaciones. Análisis estratificado por edad, sexo, estatura, índice de masa corporal y porcentaje de grasa corporal

\begin{tabular}{|c|c|c|c|c|c|c|}
\hline & Medido & Harris-Benedict & Schofield-peso & Schofield-peso/estatura & FAO/OMS & Tverskaya \\
\hline Total & $\begin{array}{c}1133 \\
(999-1355)\end{array}$ & $\begin{array}{c}1145 \\
(1079-1264)\end{array}$ & $\begin{array}{c}1181 \\
(1034-1322)\end{array}$ & $\begin{array}{c}1159 \\
(1024-1302)\end{array}$ & $\begin{array}{c}1185^{1} \\
(1089-1302)\end{array}$ & $\begin{array}{c}1230^{1} \\
(1110-1337)\end{array}$ \\
\hline \multicolumn{7}{|c|}{ 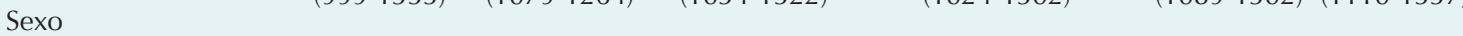 } \\
\hline Hombres & $\begin{array}{c}1212 \\
(1036-1350)\end{array}$ & $\begin{array}{c}1147 \\
(1056-1300)\end{array}$ & $\begin{array}{c}1277^{\mathrm{a}, 1} \\
(1139-1394)\end{array}$ & $\begin{array}{c}1265^{\mathrm{a}, 1} \\
(1139-1395)\end{array}$ & $\begin{array}{c}1265 \\
(1129-1379)\end{array}$ & $\begin{array}{c}1264^{\mathrm{a}, 1} \\
(1231-1369)\end{array}$ \\
\hline Mujeres & $\begin{array}{c}1098 \\
(939-1367)\end{array}$ & $\begin{array}{c}1144 \\
(1101-1262)\end{array}$ & $\begin{array}{c}1079 \\
(1016-1223)\end{array}$ & $\begin{array}{c}1087 \\
(1017-1257)\end{array}$ & $\begin{array}{c}1156^{1} \\
(1084-1278)\end{array}$ & $\begin{array}{c}1155^{1} \\
(1087-1297)\end{array}$ \\
\hline \multicolumn{7}{|l|}{ Edad (años) } \\
\hline $6-7$ & $\begin{array}{c}936^{\mathrm{b}} \\
(909-1107)\end{array}$ & $\begin{array}{c}1059^{\mathrm{b}} \\
(1008-1110)\end{array}$ & $\begin{array}{c}986^{\mathrm{b}} \\
(911-1068)\end{array}$ & $\begin{array}{c}990^{\mathrm{b}} \\
(918-1064)\end{array}$ & $\begin{array}{c}1053^{\mathrm{b}} \\
(939-1109)\end{array}$ & $\begin{array}{c}1141^{\mathrm{b}, 1} \\
(1056-1196)\end{array}$ \\
\hline $8-10$ & $\begin{array}{c}1152 \\
(1031-1368)\end{array}$ & $\begin{array}{c}1147 \\
(1123-1224)\end{array}$ & $\begin{array}{c}1161 \\
(1078-1300)\end{array}$ & $\begin{array}{c}1151 \\
(1085-1286)\end{array}$ & $\begin{array}{c}1184^{1} \\
(1139-1333)\end{array}$ & $\begin{array}{c}1221 \\
(1110-1326)\end{array}$ \\
\hline $11-12$ & $\begin{array}{c}1265 \\
(1125-1491)\end{array}$ & $\begin{array}{c}1294 \\
(1227-1390)\end{array}$ & $\begin{array}{c}1297 \\
(1223-1491)\end{array}$ & $\begin{array}{c}1304 \\
(1236-1473)\end{array}$ & $\begin{array}{c}1291 \\
(1222-1475)\end{array}$ & $\begin{array}{c}1325 \\
(1246-1404)\end{array}$ \\
\hline \multicolumn{7}{|l|}{ Estatura } \\
\hline Baja & $\begin{array}{c}1074 \\
(954-1238)\end{array}$ & $\begin{array}{c}1144 \\
(1100-1156)\end{array}$ & $\begin{array}{c}1185^{1} \\
(1043-1327)\end{array}$ & $\begin{array}{c}1190^{1} \\
(1055-1306)\end{array}$ & $\begin{array}{c}1173^{1} \\
(1116-1313)\end{array}$ & $\begin{array}{c}1172^{1} \\
(1094-1258)\end{array}$ \\
\hline Normal & $\begin{array}{c}1151 \\
(1009-1367)\end{array}$ & $\begin{array}{c}1150 \\
(1072-1265)\end{array}$ & $\begin{array}{c}1176 \\
(1022-1320)\end{array}$ & $\begin{array}{c}1156 \\
(1019-1301)\end{array}$ & $\begin{array}{c}1188^{1} \\
(1085-1326)\end{array}$ & $\begin{array}{c}1233^{1} \\
(1124-1355)\end{array}$ \\
\hline \multicolumn{7}{|c|}{ Índice de masa corporal } \\
\hline Normal & $\begin{array}{c}1086^{c} \\
(956-1259)\end{array}$ & $\begin{array}{c}1126^{c} \\
(1061-1229)\end{array}$ & $\begin{array}{c}1097^{c} \\
(993-1225)\end{array}$ & $\begin{array}{c}1098^{c} \\
(997-1244)\end{array}$ & $\begin{array}{c}1153^{c} \\
(1057-1229)\end{array}$ & $\begin{array}{c}1175^{c, 1} \\
(1090-1267)\end{array}$ \\
\hline $\begin{array}{l}\text { Sobrepeso u obesidad } \\
\% \text { grasa corporal }\end{array}$ & 1269 & 1206 & $1340^{1}$ & 1306 & $1351^{1}$ & $1319^{1}$ \\
\hline Normal & $\begin{array}{c}1094^{\mathrm{d}} \\
(954-1255)\end{array}$ & $\begin{array}{c}1126^{d} \\
(1065-1226)\end{array}$ & $\begin{array}{c}1091^{\mathrm{d}} \\
(1014-1223)\end{array}$ & $\begin{array}{c}1096^{\mathrm{d}} \\
(1013-1232)\end{array}$ & $\begin{array}{c}1149^{\mathrm{d}} \\
(1072-1229)\end{array}$ & $\begin{array}{c}11737^{\mathrm{d}, 1} \\
(1091-1258)\end{array}$ \\
\hline Obesidad & $\begin{array}{c}1361 \\
(1111-1517)\end{array}$ & $\begin{array}{c}1280 \\
(1154-1411)\end{array}$ & $\begin{array}{c}1381^{1} \\
(1240-1567)\end{array}$ & $\begin{array}{c}1353 \\
(1214-1516)\end{array}$ & $\begin{array}{c}1404^{1} \\
(1305-1548)\end{array}$ & $\begin{array}{c}1335 \\
(1240-1466)\end{array}$ \\
\hline
\end{tabular}

${ }^{1}$ Diferencias estadísticamente significativas ( $p<0.05$ en la prueba de Wilcoxon) entre el gasto energético en reposo medido y las diferentes ecuaciones.

${ }^{a}$ Diferencias estadísticamente significativas de gasto energético en reposo $(p<0.05$ por la prueba de $U$ de Mann-Whitney) por sexo. ${ }^{b}$ Diferencias estadísticamente significativas de gasto energético en reposo $(p<0.05$ en la prueba de Kruskal-Wallis) por edad. ${ }^{c}$ Diferencias estadísticamente significativas de gasto energético en reposo $(p<0.05)$ por índice de masa corporal. ${ }^{\mathrm{d}}$ Diferencias estadísticamente significativas de gasto energético en reposo $(p<0.05)$ por porcentaje de grasa corporal. 
En cuanto a las diferencias entre grupos de edad el gasto de energía en reposo aumentó significativamente con la edad; es decir, los niños de 6 a 7 años tuvieron un gasto de energía en reposo inferior al grupo de edad de 11 a 12 años. Entre los niños de 6 a 7 años el gasto de energía en reposo medido difirió de la estimación obtenida con la ecuación predictiva de Tverskaya y sus colegas (diferencia de $204.8 \mathrm{kcal} /$ día); mientras que en los niños de 8 a 9 años existieron diferencias con la estimación de las ecuaciones predictivas de la FAO/OMS (diferencias 32.1 kcal/día).

No hubo diferencias en el gasto de energía en reposo entre niños con estaturas baja y normal. Entre los niños con estatura baja cuatro de las cinco ecuaciones predictivas produjeron sobreestimaciones estadísticamente diferentes a la medida por calorimetría indirecta. En los niños con estatura baja la única estimación que no difirió con el gasto de energía en reposo medido fue la obtenida con la ecuación predictiva de Harris-Benedict. En los niños con estatura normal sólo dos ecuaciones difirieron de la medición de gasto de energía en reposo.

Comparados con niños normales, los niños con sobrepeso u obesidad medidos por índice de masa corporal y los que tenían obesidad por porcentaje de grasa tuvieron un promedio de gasto de energía en reposo medido o estimado más alto. Tanto en los niños con sobrepeso como en los obesos existieron diferencias significativas entre el gasto de energía en reposo medido y el gasto de energía en reposo derivado por la ecuación predictiva de Schofield-peso (diferencias de 70.6 y $20.0 \mathrm{kcal}$, respectivamente) y la de FAO/OMS (diferencias de 81.5 y 42.9 kcal, respectivamente). Las ecuaciones predictivas de Tverskaya y su grupo produjeron estimaciones diferentes al gasto de energía en reposo medido tanto en los niños normales de acuerdo con el índice de masa corporal (diferencia de $89.0 \mathrm{kcal}$ ) o de acuerdo con el porcentaje de grasa corporal (diferencia de $78.5 \mathrm{kcal}$ ).
El Cuadro 4 muestra la concordancia entre el gasto de energía en reposo medido y las diferentes ecuaciones predictivas. En la población total las ecuaciones predictivas de Schofield y de la FAO/OMS tuvieron la concordancia más alta $(r=0.75)$, mientras que las más bajas fueron las de Harris-Benedict y deTverskaya $(r=0.68)$. Las mismas tendencias (i.e. las concordancias más bajas se observaron con las dos últimas ecuaciones predictivas) se encontraron en las mujeres, en niños de 6 a 7 y de 8 a 10 años, en los de estatura normal, en los de índice de masa corporal normal, en los niños con sobrepeso u obesidad, en los de porcentaje de grasa corporal normal y en los que tenían obesidad. En los hombres y en el grupo de 11 y 12 años las ecuaciones predictivas de Harris-Benedict y de Tverskaya y sus colaboradores tuvieron mayor concordancia. En todas las ecuaciones predictivas la concordancia fue más baja en los niños de 6 a 7 años que en los niños de más edad.

\section{DISCUSIÓN}

En este estudio observamos que en la población total, al comparar los promedios de las estimaciones, las ecuaciones predictivas sobreestimaron el gasto de energía en reposo con diferencias especialmente altas con las ecuaciones predictivas de Tverskaya y sus colegas. En el análisis de concordancia las ecuaciones predictivas de Schofield y de FAO/OMS fueron las que tuvieron coeficientes intraclase más altos, mientras que las de Harris-Benedict y las de Tverskaya y su grupo fueron las que tuvieron el resultado más bajo.

En la muestra de escolares de la Ciudad de México, los varones de mayor edad y los que tenían sobrepeso u obesidad tuvieron mayor gasto de energía en reposo que las mujeres, los de menor edad y los que tenían peso o grasa normales. En estudios previos se observaron diferencias similares de acuerdo con el sexo, ${ }^{18-20}$ la edad ${ }^{19,21,22}$ y el estado de nutrición. ${ }^{19-23}$ En general, estas 
Cuadro 4. Coeficientes de correlación intraclase entre el gasto energético en reposo medido y las ecuaciones de predicción por sexo, estatura, índice de masa corporal y porcentaje de grasa corporal

\begin{tabular}{|c|c|c|c|c|c|c|c|c|c|c|}
\hline & \multicolumn{2}{|c|}{ Harris-Benedict } & \multicolumn{2}{|c|}{ Schofield-peso } & \multicolumn{2}{|c|}{ Schofield-peso/estatura } & \multicolumn{2}{|c|}{ FAO/OMS } & \multicolumn{2}{|c|}{ Tverskaya } \\
\hline & $r$ & $p$ & $r$ & $p$ & $r$ & $p$ & $r$ & $p$ & $r$ & $P$ \\
\hline Total & 0.68 & 0.000 & 0.74 & 0.000 & 0.74 & 0.000 & 0.74 & 0.000 & 0.68 & 0.000 \\
\hline \multicolumn{11}{|l|}{ Sexo } \\
\hline Hombres & 0.75 & 0.000 & 0.73 & 0.000 & 0.74 & 0.000 & 0.73 & 0.000 & 0.70 & 0.000 \\
\hline Mujeres & 0.60 & 0.000 & 0.76 & 0.000 & 0.76 & 0.000 & 0.75 & 0.000 & 0.66 & 0.000 \\
\hline \multicolumn{11}{|l|}{ Edad (años) } \\
\hline $6-7$ & 0.33 & 0.060 & 0.49 & 0.010 & 0.48 & 0.011 & 0.50 & 0.009 & 0.47 & 0.013 \\
\hline $8-10$ & 0.54 & 0.004 & 0.74 & 0.000 & 0.71 & 0.000 & 0.76 & 0.000 & 0.64 & 0.001 \\
\hline $11-12$ & 0.64 & 0.001 & 0.56 & 0.004 & 0.58 & 0.003 & 0.57 & 0.004 & 0.65 & 0.001 \\
\hline \multicolumn{11}{|l|}{ Estatura } \\
\hline Baja & 0.46 & 0.128 & 0.83 & 0.005 & 0.84 & 0.005 & 0.72 & 0.023 & 0.83 & 0.006 \\
\hline Normal & 0.69 & 0.000 & 0.75 & 0.000 & 0.75 & 0.000 & 0.75 & 0.000 & 0.67 & 0.000 \\
\hline \multicolumn{11}{|l|}{ Índice de masa corporal } \\
\hline Normal & 0.58 & 0.000 & 0.68 & 0.000 & 0.69 & 0.000 & 0.64 & 0.000 & 0.61 & 0.000 \\
\hline Sobrepeso u obesidad & 0.73 & 0.000 & 0.78 & 0.000 & 0.76 & 0.000 & 0.83 & 0.000 & 0.71 & 0.000 \\
\hline \multicolumn{11}{|l|}{$\%$ grasa corporal } \\
\hline Normal & 0.58 & 0.000 & 0.68 & 0.000 & 0.69 & 0.000 & 0.64 & 0.000 & 0.61 & 0.000 \\
\hline Obesidad & 0.71 & 0.001 & 0.75 & 0.000 & 0.72 & 0.001 & 0.81 & 0.000 & 0.68 & 0.000 \\
\hline
\end{tabular}

diferencias se deben a la mayor masa corporal que tiene los varones de más edad y aquellos con sobrepeso u obesidad. También pueden ser resultados de las diferencias en la composición corporal que se muestra entre sexos y edad. Los varones y los niños de mayor edad pueden tener mayor cantidad de masa libre de grasa, que es metabólicamente activa, y con ello un gasto de energía en reposo más alto. ${ }^{23}$

Una de las razones por las cuales en los escolares de la Ciudad de México las ecuaciones predictivas de Schofield y FAO/OMS tuvieron la mayor concordancia con el gasto de energía en reposo medido, es que están basadas en muestras obtenidas de diferentes áreas geográficas y de diferentes etnias. ${ }^{3,5}$ En investigaciones previas también se ha reportado adecuada concordancia de las ecuaciones predictivas de la FAO/OMS ${ }^{24}$ y de Schofield ${ }^{25}$ con calorimetría indirecta.

Aunque las ecuaciones predictivas de HarrisBenedict fueron las que tuvieron la menor diferencia con el gasto de energía en reposo medido, presentaron una concordancia menor respecto de otras ecuaciones predictivas. Este resultado se debe a que las ecuaciones fueron derivadas de población adolescente y adulta. ${ }^{7}$ Cabe señalar que en esta muestra de escolares de la Ciudad de México la concordancia fue especialmente baja en los niños de menor edad $(r=0.33)$, lo cual refuerza el hecho de que no es adecuado su uso a esa edad. ${ }^{25,26}$ En nuestra muestra se observó que la ecuación de HarrisBenedict sobrestimó el gasto de energía en reposo; sin embargo, en otra muestra de niños tendió a subestimarlo. ${ }^{21}$

Entre los niños con sobrepeso u obesidad las ecuaciones de la FAO/OMS fueron las que tuvieron la mayor concordancia, seguidas por las de Schofield. A pesar de que las ecuaciones predictivas de Tverskaya y sus colaboradores fueron desarrolladas en niños con obesidad, su concordancia en los niños con esta característica fue menor respecto a las dos ecuaciones predictivas anteriores. Contrario a lo reportado en otra muestra de escolares mexicanos, ${ }^{21}$ donde se 
observó que la ecuación predictiva de Tverskaya subestimaba el gasto de energía en reposo, en nuestra muestra lo sobrestimaban. En cualquier caso, las ecuaciones predictivas de Tverskaya tiene la limitante adicional de requerir mediciones de composición corporal que no siempre están disponibles; mientras que para usar las otras dos ecuaciones sólo se necesita la información más sencilla de compilar (i.e. peso y estatura).

Los niños con sobrepeso u obesidad tuvieron mayor gasto de energía en reposo, lo cual es atribuible a que tienen más masa corporal. Las diferencias en gasto de energía entre personas obesas y con peso normal desaparecen cuando el gasto se expresa por kilogramo de peso. ${ }^{27}$

Considerando que en escolares brasileños la estatura baja se relacionó con menor gasto de energía en reposo y mayor ganancia de peso; ${ }^{28} \mathrm{se}$ esperaba que las ecuaciones predictivas fueran menos exactas en escolares con estatura baja. Sin embargo los resultados en los escolares de la Ciudad de México fueron inconsistentes. Por un lado, en los niños con estatura baja hubo diferencias entre el gasto de energía en reposo estimado con cuatro ecuaciones predictivas (las dos de Schofield, la de FAO/OMS y la de Tverskaya) con el gasto de energía en reposo medido; en los de estatura normal existieron diferencias con dos ecuaciones predictivas. Por otro lado, la concordancia entre tres ecuaciones predictivas y el gasto de energía en reposo medido fue mayor en los niños con estatura baja que en los de estatura normal. Conviene señalar que en estudios previos en preescolares brasileños ${ }^{29}$ y en escolares mexicanos ${ }^{30}$ no se han mostrado diferencias en el gasto de energía en reposo entre niños con y sin estatura baja. Se requiere más investigación para conocer si la estimación del gasto de energía en reposo en niños con desnutrición crónica puede realizarse con ecuaciones predictivas desarrolladas para población general o si se deben establecer ecuaciones específicas.
Entre las limitaciones de este estudio se encuentra el tamaño reducido de la muestra, especialmente de niños con estatura baja, por lo cual se requiere más investigación sobre el posible efecto de la desnutrición crónica en el gasto de energía en reposo. Se utilizó una muestra por conveniencia, lo cual limita la posibilidad de extrapolar los resultados a otros grupos de niños. Además, se ha cuestionado que el índice de masa corporal sea un indicador apropiado de adiposidad en escolares mexicanos.

\section{CONCLUSIONES}

Aunque se requiere más investigación sobre la conveniencia de usar ecuaciones de predicción del gasto de energía en reposo en población pediátrica de México, nuestros resultados apoyan el uso de las ecuaciones de la FAO/OMS o las de Schofield.

\section{AGRADECIMIENTOS}

Agradecemos a los niños que aceptaron participar en el estudio y a la licenciada en nutrición Magdalena Rodríguez Magallanes (responsable de la Unidad de Nutrición, Composición Corporal y Gasto de Energía de la Licenciatura en Nutrición Humana, Universidad Autónoma Metropolitana Xochimilco) por su apoyo para la realización del trabajo de campo.

\section{REFERENCIAS}

1. Gutiérrez JP, Rivera-Dommarco J, Shamah-Levy T, VillalpandoHernández S, Franco A, Cuevas-Nasu L, et al. Encuesta Nacional de Salud y Nutrición 2012. Resultados Nacionales. Cuernavaca, México: Instituto Nacional de Salud Pública, 2012.

2. FAO/WHO/UNU. Human energy requirements: report of a joint FAO/WHO/UNU Expert Consultation. Food Nutr Bull 2005;26(1):166.

3. Schofield WN. Predicting basal metabolic rate, new standards and review of previous work. Hum Nutr Clin Nutr 1985;39(1):5-41.

4. Das SK. Metabolismo energético. En: Browman BA, Russell $R$, editores. Conocimientos actuales sobre nutrición. 
Washington DC: Organización Panamericana de la Salud, Instituto Internacional de Ciencias de la Vida, 2003.

5. FAO/WHO/UNU. Energy and protein requirements. Report of a joint FAO/WHO/UNU Expert Consultation. World Health Organization Technical Report Series no. 724, 1985.

6. Tverskaya R, Rising R, Brown DFL. Comparison of several equations and derivation of a new equation for calculating basal metabolic rate in obese children. J Am Coll Nutr 1998;17(4):333-8.

7. Harris JA, Benedict FG. A biometric study of human basal metabolism. Proc Natl Acad Sci USA 1918;4(12):370-3.

8. Simal A. Balance Energético. En: Hernández M, Sastre A, editores. Tratado de nutrición. Madrid: Edición Díaz de Santos; 2006:589-600.

9. Matarese LE. Indirect calorimetry: technical aspects. J Am Diet Assoc 1997;97(10 Suppl 2):S154-60.

10. Compher C, Frankenfield D, Keim N, Roth-Yousey L, Evidence Analysis Working G. Best practice methods to apply to measurement of resting metabolic rate in adults: a systematic review. J Am Diet Assoc 2006;106(6):881-903.

11. Weir V. New methods for calculating metabolic rate with special reference to protein metabolism. J Physiol 1949;109:1-9.

12. Lohman TG, Roche AF, Martorell R. Anthropometric Standarization Reference Manual. Champaign, IL: Human Kinetics; 1988.

13. Habicht J. Estandarización de métodos epidemiológicos sobre el terreno. Bol Of Sanit Panam 1974;76:375-84.

14. de Onis M, Onyango AW, Borghi E, Siyam A, Nishida C, Siekmann J. Development of a WHO growth reference for school-aged children and adolescents. Bull World Health Organ 2007;85(9):660-7.

15. Slaughter MH, Lohman TG, Boileau RA, Horswill CA, Stillman RJ, Van Loan MD, et al. Skinfold equations for estimation of body fatness in children and youth. Hum Biol 1988;60(5):709-23.

16. Mirwald RL, Baxter-Jones AD, Bailey DA, Beunen GP. An assessment of maturity from anthropometric measurements. Med Sci Sports Exerc 2002;34(4):689-94.

17. Williams DP, Going SB, Lohman TG, Harsha DW, Srinivasan SR, Webber LS, et al. Body fatness and risk for elevated blood pressure, total cholesterol, and serum lipoprotein ratios in children and adolescents. Am J Public Health 1992;82(3):358-63.

18. Goran MI, Nagy TR, Gower BA, Mazariegos M, Solomons $\mathrm{N}$, Hood V, et al. Influnce of sex, seasonality, ethnicity and geographic location on the components of total energy expenditure in young children: implications for energy requierements. Am J Clin Nutr 1998;68:675-81.

19. Tershakovec A, Kuppler KM, Zemel B, VA. S. Age, sex, etnicity, body composition, and resting energy expenditure of obese African American and White children and adolescents. Am J Clin Nutr 2002;75:867-71.

20. Paz M, Sierra C, Mapelli L, Abarco A, Delgado C, Jurado Al. Influencia del gasto energético en la obesidad infantil. An Pediatr 2003;58(4):316-21.

21. Balas-Nakash $M$, Villanueva-Quintana $A$, Vadillo-Ortega $F$, Perichart-Perera O. Validación del uso de fórmulas para estimar el gasto energético en reposo en niños mexicanos de 9 a 12 años de edad con y sin obesidad. Rev Invest Clín 2008;60(5):395-402.

22. Azcona CFG. Gasto energético en niños y adolescentes sanos y afectados de obesidad. Rev Esp Obes 2009;7(1):52-8.

23. DeLany J, Bray GA, Harsha DW, Volaufova J. Energy expenditure in preadolescent African American and White boys and girls: the Baton Rouge Children's study. Am J Clin Nutr 2002;75:705-13.

24. Henry CJK, Dyer S, Ghusain-Choueiri A. New equations to estimate basal metabolic rate in children aged $10-15$ years. Eur J Clin Nutr 1999;53:134-43.

25. Derumeaux BH, Meyer M, Morin L, Boirie Y. Prediction of resting energy expenditure in a large population of obese children. Am J Clin Nutr 2004;80:1544-50.

26. García P, Bullo M, Salas J. El cuerpo humano: técnicas de estudio del consumo energético. En: Miján A, editor. Técnicas y métodos en investigación humana. Barcelona: Glosa Editores, 2002:165-182.

27. Carpenter WH, Poehlman ET, Oconnell M, Goran MI. Influence of body-composition and resting metabolic-rate on variation in total-energy expenditure -a metaanalysis. Am J Clin Nutr 1995;61(1):4-10.

28. Grillol LP, Siqueira AF, Silva AC, Martins PA, Verreschi IT, Sawaya AL. Lower resting metabolic rate and higher velocity of weight gain in a prospective study of stunted vs nonstunted girls living in the shantytowns of Sao Paulo, Brazil. Eur J Clin Nutr 2005;59(7):835-42.

29. Hoffman DJ, Sawaya AL, Coward WA, Wright A, Martins PA, de Nascimento $C$, et al. Energy expenditure of stunted and nonstunted boys and girls living in the shantytowns of Sao Paulo, Brazil. Am J Clin Nutr 2000;72(4):1025-31.

30. Wilson HJ, Dickinson F, Hoffman DJ, Griffiths PL, Bogin B, Varela-Silva MI. Fat free mass explains the relationship between stunting and energy expenditure in urban Mexican Maya children. Ann Human Biol 2012;39(5):432-9. 